\title{
White-coat and masked hypertension and coronary artery disease: are they related or not?
}

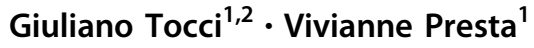

Received: 12 October 2019 / Revised: 2 November 2019 / Accepted: 2 November 2019 / Published online: 29 November 2019

(c) The Japanese Society of Hypertension 2019

Currently, high blood pressure (BP) may cover different hypertension phenotypes, including white coat hypertension (WCHT), masked hypertension (MHT), and sustained hypertension (SHT), all of which are characterized by substantially higher risk of cardiovascular (CV) events and mortality than normotension (NT) [1-5]. This has recently been reaffirmed by the latest sets of European guidelines [6], which emphasized the role of out-of-office BP assessment, such as 24-h ambulatory and home BP monitoring, to properly stratify apparently low-risk individuals with highnormal BP levels. The same guidelines also recommended that a search for hypertension-mediated organ damage or other risk factors and comorbidities should be performed in patients with WCHT or MHT, in view of the relative frequency of these risk factors and markers of organ damage in these hypertensive patients [6].

Despite this evidence, both WCHT and MHT are often undiagnosed or even untreated. Findings from large national databases or clinical studies have clearly demonstrated that both of these conditions are relatively common in the clinical practice of hypertension [7]. Other studies have also reported that, even in the presence of antihypertensive treatment, effective BP control is not achieved in patients with WCHT or MHT, with obvious and relevant consequences on the risk of developing major $\mathrm{CV}$ outcomes [8-10].

Sustained BP elevation still represents the major contributing factor for the occurrence of coronary events, including acute coronary syndrome, myocardial infarction,

Giuliano Tocci

giuliano.tocci@uniroma1.it

1 Hypertension Unit, Division of Cardiology, Department of Clinical and Molecular Medicine, Faculty of Medicine and Psychology, Sant'Andrea Hospital, University of Rome Sapienza, Rome, Italy

2 IRCCS Neuromed, Pozzilli, IS, Italy coronary revascularization, and death due to coronary disease [11]. Thus, it can be argued that early identification of patients with uncontrolled BP levels and asymptomatic coronary artery disease might improve $\mathrm{CV}$ disease prevention and reduce the burden of hypertension-related $\mathrm{CV}$ morbidity and mortality.

In this issue of Hypertension Research, Wang and coworkers present an analysis of a relatively large database of adult hypertensive outpatients with symptoms of angina and electrocardiographic changes of myocardial ischemia, who were stratified in the four hypertension phenotypes. All patients underwent coronary angiography and conventional electrocardiogram to assess the presence of coronary artery stenosis or cardiac arrhythmias, respectively. In this population, the prevalence of MHT was remarkably higher than, while the prevalence of WCHT was similar to, that reported in previous clinical studies performed in different clinical settings, as schematically shown in Fig. 1 [1-5].

The prevalence of different hypertension phenotypes may be affected by several factors, such as BP thresholds, inclusion/exclusion criteria for the reference population, and the reproducibility of BP assessments over time [12, 13]. In the study performed by Wang, the observed high prevalence of MHT can be explained by the very high-risk profile of patients with the signs or symptoms of myocardial ischemia. On the other hand, the observed prevalence of MHT can also be conditioned by the BP thresholds used for the definitions of the different hypertension phenotypes. Indeed, Wang and coworkers adopted clinic and all ambulatory (24-h, day-time and night-time) BP thresholds for identifying WCHT and MHT, while other authors used the combination of clinic and either home or 24-h ambulatory BP levels.

Beyond these considerations, all these studies agreed on the fact that both WCHT and MHT are associated with a higher risk of CV outcomes than NT [1-5]. Of note, in the study by Wang and coworkers, the incidence of coronary artery disease in both the MHT and SHT groups were significantly higher than that in the NT group, but there was no 
Fig. 1 Prevalence of different hypertension phenotypes in different clinical settings.

Derived from the refs. [1-5]

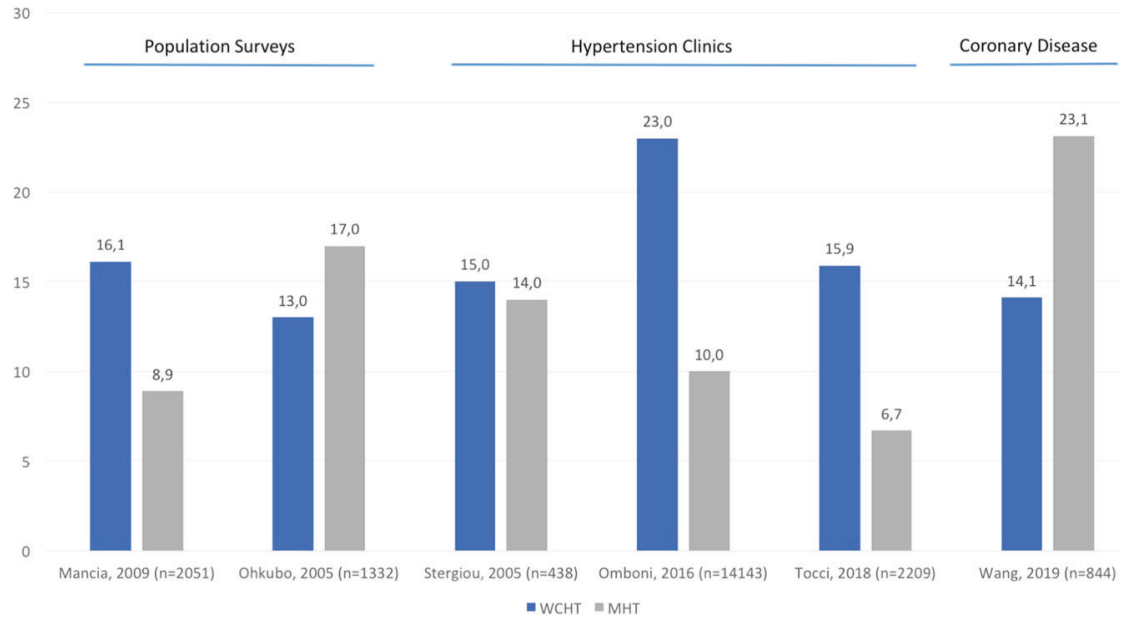

significant difference between the WCHT and the NT groups, thus highlighting that neither of these conditions should be considered an innocent report.

In conclusion, based on the available evidence, integrated clinical risk assessment should be adopted in patients with either WCHT or MHT to prevent the occurrence of major CV events, mostly coronary artery disease.

Early recognition of these individuals is a key element for adopting preventive strategies aimed at reducing their $\mathrm{CV}$ risk profile, as also highlighted by current European guidelines [6]. Thus, more widespread use of out-of-office BP measurements might also be recommended in the general population [6], with the aim of identifying those patients with WCHT or MHT before the development of SHT or the occurrence of major CV outcomes.

\section{Compliance with ethical standards}

Conflict of interest The authors declare that they have no conflict of interest.

Publisher's note Springer Nature remains neutral with regard to jurisdictional claims in published maps and institutional affiliations.

\section{References}

1. Tocci G, Presta V, Figliuzzi I, Attalla El Halabieh N, Battistoni A, Coluccia R, et al. Prevalence and clinical outcomes of white-coat and masked hypertension: analysis of a large ambulatory blood pressure database. J Clin Hypertens. 2018;20:297-305.

2. Mancia G, Bombelli M, Facchetti R, Madotto F, Quarti-Trevano F, Polo Friz H, et al. Long-term risk of sustained hypertension in white-coat or masked hypertension. Hypertension. 2009;54: 226-32.
3. Ohkubo T, Kikuya M, Metoki H, Asayama K, Obara T, Hashimoto J, et al. Prognosis of "masked" hypertension and "whitecoat" hypertension detected by 24-h ambulatory blood pressure monitoring 10-year follow-up from the Ohasama study. J Am Coll Cardiol. 2005;46:508-15.

4. Stergiou GS, Salgami EV, Tzamouranis DG, Roussias LG. Masked hypertension assessed by ambulatory blood pressure versus home blood pressure monitoring: is it the same phenomenon? Am J Hypertens. 2005;18:772-8.

5. Omboni S, Aristizabal D, De la Sierra A, Dolan E, Head G, Kahan $\mathrm{T}$, et al. Hypertension types defined by clinic and ambulatory blood pressure in 14143 patients referred to hypertension clinics worldwide. Data from the ARTEMIS study. J Hypertens. 2016; 34:2187-98.

6. Williams B, Mancia G, Spiering W, Agabiti Rosei E, Azizi M, Burnier M, et al. $2018 \mathrm{ESC} / \mathrm{ESH}$ guidelines for the management of arterial hypertension. Eur Heart J. 2018;39:3021-104.

7. Nagasawa Y, Kida A, Hasuike Y, Kuragano T, Nakanishi T. White-coat hypertension and albuminuria; lessons from the Hisayama and Ohasama studies. Hypertens Res. 2018;41:483-5.

8. Fujiwara T, Matsumoto C, Asayama K, Ohkubo T, Hoshide S. Are the cardiovascular outcomes of participants with white-coat hypertension poor compared to those of participants with normotension? A systemic review and meta-analysis. Hypertens Res. 2019;42:825-33.

9. Siven SS, Niiranen TJ, Kantola IM, Jula AM. White-coat and masked hypertension as risk factors for progression to sustained hypertension: the Finn-Home study. J Hypertens 2016;34:54-60.

10. Satoh M, Asayama K, Murakami T, Kikuya M, Metoki H, Imai Y, et al. Stroke risk due to partial white-coat or masked hypertension based on the ACC/AHA guideline's blood pressure threshold: the Ohasama study. Hypertens Res. 2019;42:120-2.

11. Lewington S, Clarke R, Qizilbash N, Peto R, Collins R. Agespecific relevance of usual blood pressure to vascular mortality: a meta-analysis of individual data for one million adults in 61 prospective studies. Lancet. 2002;360:1903-13.

12. Asayama K, Satoh M, Kikuya M. Diurnal blood pressure changes. Hypertens Res. 2018;41:669-78.

13. Mancia G, Facchetti R, Cuspidi C, Bombelli M, Corrao G, Grassi G. Limited reproducibility of MUCH and WUCH: evidence from the ELSA study. Eur Heart J. 2019. [Epub ahead of print]. 